\title{
Design Methodology for FPGA Implementation of Lattice Piecewise-Affine Functions
}

M. C. Martínez-Rodríguez², I. Baturone ${ }^{1,2}$, P. Brox ${ }^{2}$

Department of Electronics and Electromagnetism, University of Seville Microelectronics Institute of Seville (IMSE-CNM-CSIC) Seville, Spain December, 2011

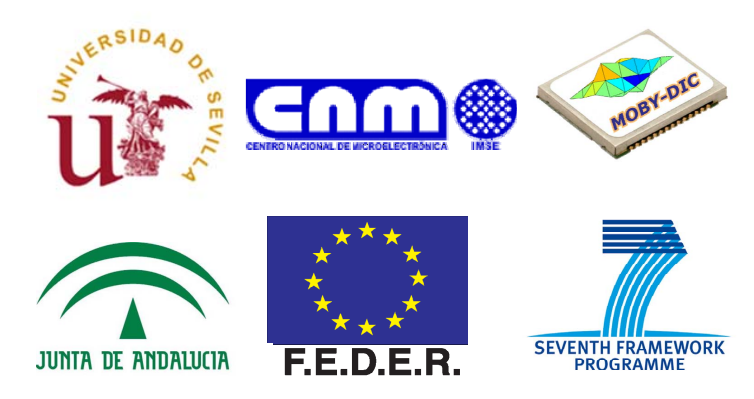




\section{Design Methodology for FPGA Implementation of Lattice Piecewise-Affine Functions}

- A piecewise-affine (PWA) function provides a linear (affine) output for each region in which the input domain, $\mathrm{D}$, is partitioned.

- The lattice approach [1]

- allows the implementation of any continuous PWA function.

- selects the affine function without evaluating the boundaries.

- The algorithm extracts the lattice PWA representation using:

- a simplied structure matrix $\rightarrow$ relation between regions

- A simplied parameter $\rightarrow$ affine regions 


\section{Design Methodology for FPGA Implementation of Lattice Piecewise-Affine Functions}

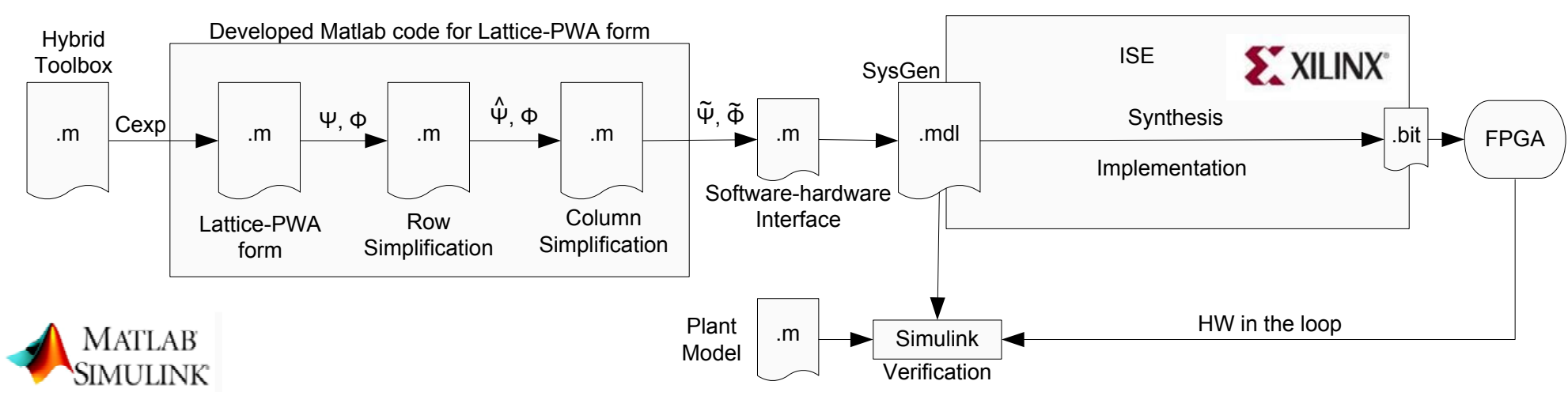

- The design methodology presented for FPGA implementation of continuous PWA functions based on lattice representation has been automated with Matlab\&Simulink and ISE tools.

- The parameters required by the digital architecture are obtained from the algorithmic description of the problem.

- FPGA implementation results for applications in the control domain offer small size, high speed, and potentially no approximation error with regards to the optimum solution. 